\title{
An Innovative and Interactive Approach to Teaching Industrial Drawing to Engineering Students
}

\author{
André Cincou, ing., M.Sc.A. \\ Department of Mechanical Engineering \\ Design and Manufacturing Section \\ Polytechnique Montréal \\ University of Montreal \\ Montreal H3T 1J4, Quebec, Canada \\ email: andre.cincou@polymtl.ca
}

\begin{abstract}
This paper presents an innovative and interactive approach to teaching industrial drawing methods, which is designed to improve the way students learn about the spatial visualization of objects by means of orthographic projection and the resolution of complex orthographic projection problems, and how they master the standards and conventions of graphical representation.

This new approach is supported by two innovative tools that function in accordance with the standards and prescribed approaches of the Bachelor of Engineering degree program at Polytechnique Montréal, and was validated in a classroom setting.

The products of this research are a $2 D / 3 D$ multimedia tutorial and a guide to the standards and conventions related to graphical representation which supports the tutorial.

The examples provided in the tutorial teach students to visualize, with all the advantages of a multimedia presentation, a strategy for resolving orthographic projection problems. They are supported by the geometric laws that form the foundation of the resolution strategy itself. The strategy is based on the principles and techniques that are taught to students to develop their spatial visualization capabilities, in the belief that better visualization of those basic laws will allow students to fully grasp the nature of $3 D$ objects in space, and obviate the need to apply a step-by-step resolution strategy.

The tutorial is a tool that is supported by a reference document which outlines the standards and conventions governing graphical representation for product definition. This document is mainly composed of illustrations of examples and counterexamples which are accompanied by comments or brief descriptive paragraphs. This document is exhaustive and up to date, and, most importantly, it follows the standards prescribed by ACNOR CAN3-78-1-M83 and ISO 128-20.
\end{abstract}

Used together, the tutorial and the guide save teachers time in the classroom, as well as facilitating the integration of new concepts into the course material for training future engineers, which have been approved by Polytechnique Montréal and the industry.

The tutorial and guide also enable students to learn the various concepts more quickly, help them become more autonomous, and provide them with a structured method for finding valid solutions to complex orthographic projection problems.

Keywords: industrial drawing, technical drawing, engineering graphics, multimedia, tutorial, geometric laws, orthographic projection problems, standards and conventions, graphical representation, spatial visualization.

\section{INTRODUCTION}

The aim of this project is to improve the quality of student learning in industrial (technical) drawing, initially for future engineers being trained at Polytechnique Montréal, and subsequently for students at Cégeps and technical schools.

Industrial drawing is a technique for characterizing a product using a graphical language governed by established standards and conventions. The methods used to express this language are freehand drawing (sketching), traditional drawing using appropriate tools, and computer aided design (CAD).

Teaching this material is a challenge, particularly when the objective is to resolve the complex problems of orthographic projection. This is because of the difficulty of clearly illustrating this material using the conventional aids available to teachers, such as blackboards, transparencies, slides, paper, etc. Also, this type of course content is assimilated in very different ways by students, who don't all have the same level of knowledge to begin with. For some, a blackboard demonstration is sufficient 
to master the fundamental principles required to resolve an orthographic projection problem, while others need to see the demonstration, or part of it, a number of times to reach the same level of learning. However, repeating these demonstrations in the classroom is not practical, nor is it effective, as resolving this type of problem involves more than simply intuitively visualizing an object in 3D. Principles, techniques, mechanisms, and a structured approach are required to enable students to mentally construct a 3D representation from a 2D rendering of an object, and to acquire the necessary skills to learn CAD or CAO.

The use of multimedia resources (iconography, animation, direct image manipulation, videography, etc.) can be immensely helpful in facilitating comprehension of the concepts related to spatial visualization. Animation, for example, makes it possible to move forward or back, or pause, in a demonstration, or to repeat any part of the presentation. With the 3D feature of a multimedia tool, image manipulation can be illustrated in a true 3D environment, without interference from the optical illusions that can be generated in a 2D medium.

The course manual that traditionally forms the basis for lectures in industrial drawing at Polytechnique Montréal dates from the 1980s, and contains much more content than is included in the course.

Assessments of the quality of the teaching of the course completed by large groups of students show overall satisfaction. However, some facets of the course, such as lectures recommended to students to help them better understand the material, regularly achieve significantly lower scores. In general, the students consider that the manual, while a useful reference, is poorly structured and difficult to navigate. They also consider that it needs to be updated.

We develop two tools in this work to achieve the objectives of our research: 1) an up-to-date reference work designed to provide an overall view of the topic, which takes a new approach to presenting the standards and conventions of graphical representation and problem resolution; and 2) educational software to accompany the reference work aimed at giving students an opportunity to learn the theoretical concepts more quickly, to engage in self-teaching, and to adopt a structured approach to analyzing orthographic projection problems.

\section{METHODS}

\subsection{Standards guide}

The first part of our proposed solution involves putting together a reference work containing the standards and conventions governing graphical representation in the product definition process.
This guide is primarily composed of figures illustrating examples and counterexamples. Short texts accompany the figures.

We intend the document to provide an overall view of the subject, to be up to date, and, most importantly, to respect the following standards: ACNOR CAN3-78-1M83 [1], CAN/CSA-78-2-M91 [2], and ISO 128-20 [8].

\subsection{Educational software}

The second part of our proposed solution involves the production of multimedia educational software to accompany the standards guide.

Using the many advantages of multimedia applications, our educational software sets out a strategy for resolving orthographic projection problems. It provides a number of examples and details the fifteen basic principles (rules of geometry) that constitute the foundation for this resolution strategy.

The standards and conventions governing graphical representation in special cases are also addressed, in five additional modules.

This content is presented by means of a number of graphics windows that can be expanded or contracted, as the user wishes.

The software also includes an interactive help module, which is available to the user at all times.

Adobe Flash has been used to generate the 2D and 3D animations for the software. This technology, which was launched in 1996, has become very popular among developers for creating animations and interactive objects, its major advantage being that most operating systems are capable of reading Flash content without difficulty. Moreover, because the technology is so widely deployed on the Web, it is safe to assume that Flash will continue to be in use in the short term and well into the medium term, and possibly even beyond, which will help to ensure a long life for our software.

Moodle, a website dedicated to teaching management, is the host for this software, which can be run independently of the operating system (Windows, Mac OS $\mathrm{X}$, Linux, Unix) or Web browser used (Internet Explorer, Netscape, Safari, Firefox).

\subsection{Assessing the usefulness of the new teaching modalities}

In order to gauge the usefulness of the standards guide and the educational software, we sought the opinions of three groups of principal actors who have been involved with the following courses in some way: ING1020 (Graphic communication), MEC1515 (CAD in engineering), and MEC1510 (Modeling mechanical systems). They are: the principal author of the research and this 
paper, the teachers of the courses concerned, and students enrolled in these courses respectively.

The author and a group of four teachers were in a position to assess the quality of the teaching of industrial drawing courses before and after the implementation of the new teaching aid and method. The students were also invited to evaluate the courses.

We triangulated the accounts of the experiences of the individuals in the three principal groups of actors, in order to gauge the impact of the new teaching modalities.

\section{STANDARDS GUIDE}

\subsection{Guide content}

The Standards Guide [4] consists of six chapters and an appendix. The chapter headings are listed as follows:

- Drawing conventions and practices;

- Conventional shape description;

- Sectional views and conventions;

- Dimensioning;

- Thread symbols and thread notes;

- Design and working drawings;

- Appendix.

\subsection{Layout and structure}

A typical page is composed of an illustration area (which takes up at least $75 \%$ of the available space) and an area (the right-hand margin) for note taking (see Fig. 1).

The illustration captions are formatted in italics, and the text related to the drawings (titles, dimensions, and notes) is formatted in standard capital letters.

We illustrate each theme presented using examples or counterexamples.

In rare cases ( 5 pages out of 50 ), the note-taking area is reduced to accommodate a definition or interpretation.

An important feature of the Standards Guide is that all the information relating to a theme can be found on a single page. This means that readers can focus their attention in one place, instead of having to look through several pages to find the information they are seeking: one theme, one page.

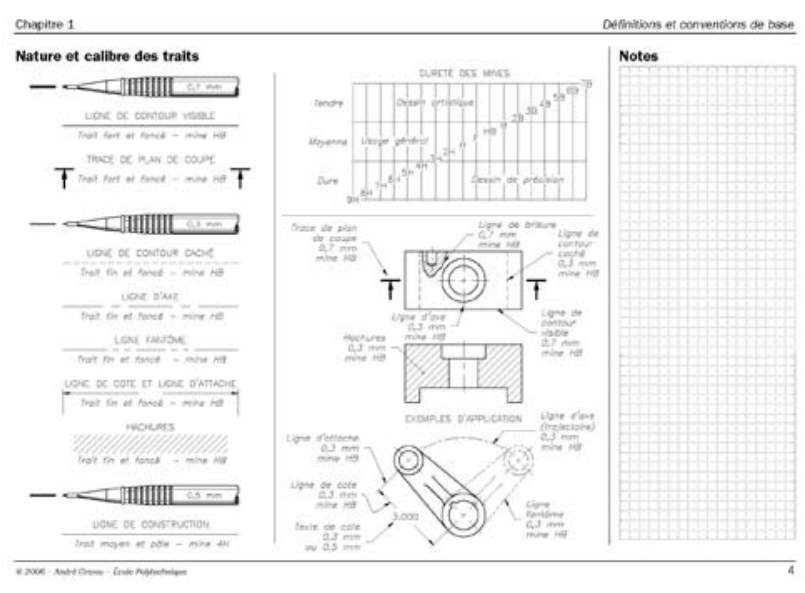

Fig. 1. A typical page in the Standards Guide [4].

\section{EDUCATIONAL SOFTWARE}

\subsection{Software content}

The content of the software [3] is presented in three sections, as follows:

1. The principles, conventions, and rules of geometry on which the problem resolution strategy is based.

2. Six detailed examples of problem resolution using the problem resolution strategy.

3. The conventions governing graphical representation in special cases.

\subsection{Interface and structure}

When the software is started, the welcome screen displays the Menu window, which allows the user to choose one of the five available tools (see Fig. 2).

The window tabs Menu and Basic principles are accessible at all times, whatever tool the user has selected.

Clicking on the Basic principles tab, which is located at the bottom right of the interface, enables the user to choose to view any of the principles and geometric rules on which orthographic projection problem resolution is based. It serves as a kind of dictionary that is readily, and permanently, available to users.

Clicking on the Menu tab on the welcome screen offers the user the choice of viewing any one of the six examples provided of problem resolution. Once a selection has been made, the Resolution window partially opens. Additional information can be displayed by expanding the window vertically, using the drag-and-drop feature. The problem name appears in the upper left corner of the screen, the instructions are displayed in the work space, and the tabs for opening the $3 \mathrm{D}$ and isometric views of the object 
under study are located in the upper right corner of the screen.

The interfaces for the five modules under the heading Drawing conventions are similar. The various sections of a module can be consulted independently, by tapping the corresponding buttons on the Controls tab. The sections can be viewed sequentially by clicking on the arrows at the right or left end of the Controls tab (see Fig. 3).

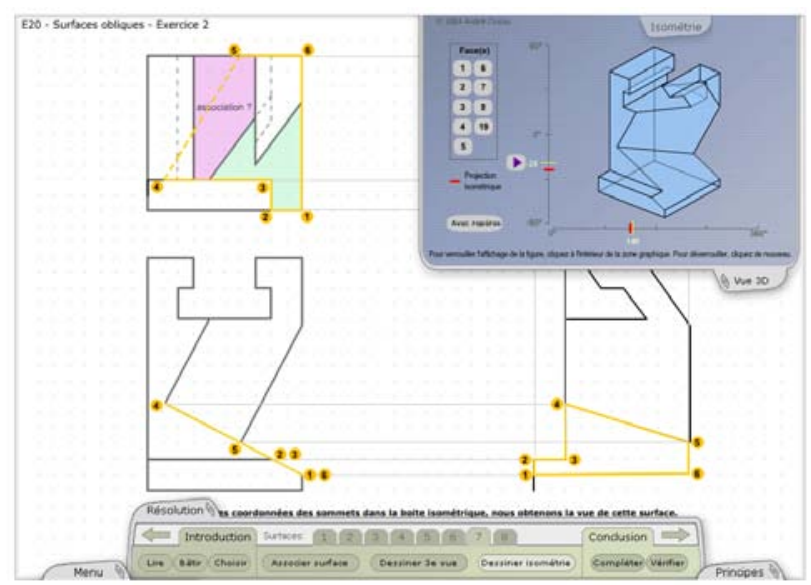

Fig. 2. Resolution window [3].

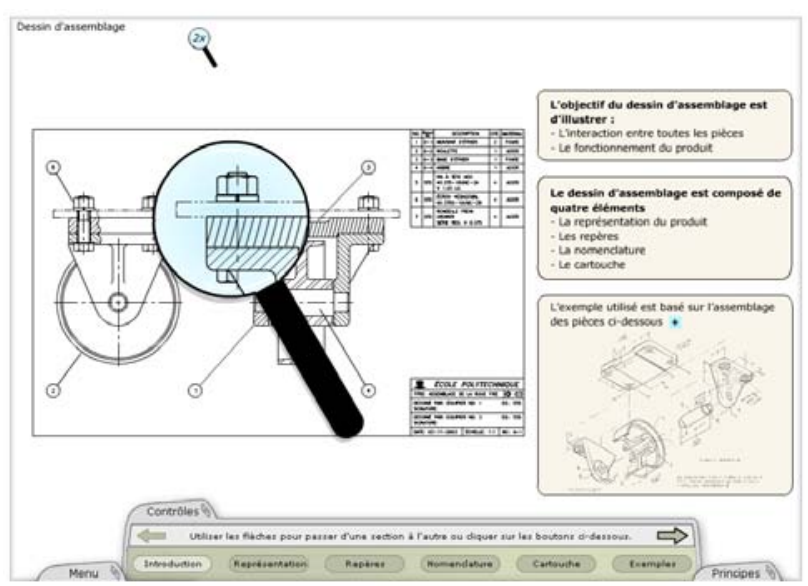

Fig. 3. Drawing conventions interface [3].

\section{ASSESSING THE USEFULNESS OF THE NEW TEACHING AID AND METHOD}

We assessed the usefulness of the new teaching aid and method using three tools: (1) assessment by the author; (2) assessment by teachers with knowledge of the teaching context before and after implementation of these new teaching modalities; and (3) a survey, by means of a questionnaire, conducted among users who are students enrolled in courses MEC1510 and MEC1515. The first two of these tools are experience-based.

\subsection{Synthesis of the teachers' accounts of their experiences}

An examination of the teachers' assessments overall reveals what their experiences with the Standards Guide and the educational software have in common, and what they consider to be the impact of the new teaching modalities. These shared viewpoints are presented in sections 5.1.1 to 5.1.3.

\subsubsection{Standards Guide}

- The content on the standards is up to date, and organized to correspond to the presentation of the course material.

- The information presented is limited to the elements required to teach the course.

- With the Guide complementing the software, teachers are able to cover the theory more quickly, leaving time available to teach other concepts, such as reading drawings and using tools like CAD and CAO.

- The format of the Guide is practical and cost effective.

- There is scope to enhance the content of the Guide in the future; for example, adding themes to expand the range of industrial drawing as a subject taught in an educational context.

\subsubsection{Educational software}

- The software enables students to visualize concepts in 3D.

- Student can learn at their own pace by viewing the demonstrations presented in class as many times as they wish.

- Teachers can create a more powerful synthesis of the concepts they are teaching.

- It is well adapted to the needs of students.

\subsubsection{Impact of the new teaching modalities}

- Students retain the course material more effectively.

- Less time is invested by students, with no compromise in the quality of the training they receive.

- With the Guide as a complement, the software accelerates the pace at which basic concepts can be learned, and, consequently, increases the rate of introduction of new material. 


\subsection{Synthesis of the student survey results}

The course assessments for ING1020, which was given between the fall of 2001 and the winter of 2004, show that question 5 (on whether the lectures recommended to help students learn the material more effectively achieve that objective) and question 6 (on whether the mandatory purchase of printed materials for the course is justified) both generally earned weaker rates of agreement among the students surveyed.

In an effort to identify the source of the problem, another survey was conducted, this time among students enrolled in the course in the winter 2004 semester.

The questionnaire covered several documents used in the course, including the reference manual [6]. The results of the survey show that the problem with the manual was related to its clarity, usefulness, and frequency of use.

To determine the impact of replacing this manual with the Standards Guide, a similar survey, targeting clarity, usefulness, and frequency of use, was conducted among students who had used the Guide between the fall of 2006 and the fall of 2007.

The results of this survey were compared with the results of the winter 2004 survey.

The comparison shows that, in terms of clarity, the Standards Guide represents a mean improvement of $27 \%$ over the reference manual. The same is true for the usefulness of the Guide, with a mean improvement of $35 \%$. Finally, with respect to the frequency of use of the reference manual during the winter of 2004 compared to that from the fall of 2006 to the fall of 2007, the results show that $36 \%$ of the students rarely use the manual, $46 \%$ of them use it sometimes, and $18 \%$ of them use it regularly. The results also indicate that $6 \%$ of students rarely use the Guide, $45 \%$ of them use it sometimes, and $49 \%$ of them use it regularly.

The frequency of regular use of the manual in the course jumped by $31 \%$ following the introduction of the Standards Guide. By contrast, the percentage of students who only use the manual sometimes remains virtually unchanged. We hypothesize that these students had understood the concepts from the classroom presentations.

Finally, the results show that the dissatisfaction of the students with using and purchasing printed reference materials diminished with the addition of a work that better serves their needs.

Figure 4 and Figure 5 show the percentages of students who agree and disagree with the statement in question 5 (the recommended lectures help students learn the material more effectively) and question 6 (the mandatory purchase of printed materials for the course is justified) of the course assessment questionnaire respectively. These figures also present the means of the percentages of students who disagree with the statements, by plateau, with respect to the following three reference works:
- A: Giesecke et al.'s technical drawing textbook [5];

- B: Giesecke et al.'s technical drawing textbook [6] [7];

- C: Cincou’s Standards Guide.

From these results, we can conclude that the dissatisfaction of students with using and purchasing reference materials diminishes with the addition to the course of a work that better serves their needs.

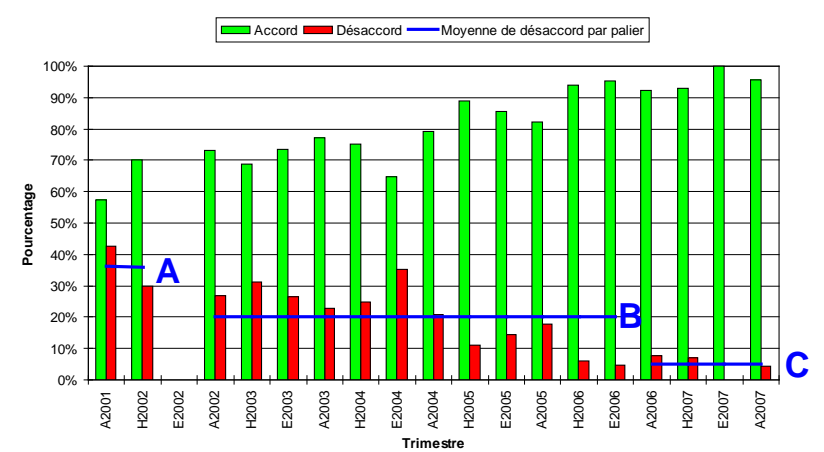

Fig. 4. Percentage of agreement and disagreement, and the mean disagreement by plateau, for question 5 of the course assessment, with respect to the three reference works used: A [5], B [6][7], and C [4].

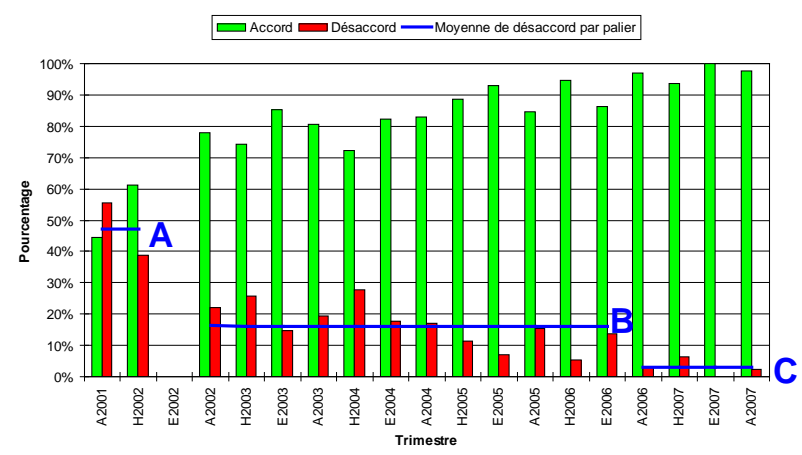

Fig. 5. Percentage of agreement and disagreement, and the mean agreement and mean disagreement by plateau, for question 6 of the course assessment questionnaire, with respect to the three reference works used: A [5], B [6][7], and C [4].

\section{CONCLUSIONS}

With this research work, we have achieved the following:

- Improved the quality of industrial drawing training for engineering students at Polytechnique Montréal.

- Reduced the amount of time teachers spend in the classroom, enabling them to integrate new concepts into their courses in response to the expectations of 
the industry in terms of the training of future engineers.

- Reduced the amount of time required by students to learn the theoretical concepts, and provided them with a structured approach for the analysis of orthographic projection problems.

- Enabled students to engage in self-teaching during the training process.

These new teaching modalities enable us to more effectively teach our engineering students two indispensable skills that they must acquire during their training:

1. Analysis and interpretation of the definition of an object, with the ultimate objective of spatial visualization of the object.

2. Mastery of the principles and standards of graphical representation that enable the bidirectional transfer of data: either to define a product in 2D from a 3D model, or, conversely, to generate a 3D model from a 2D sketch.

The originality of this work lies in the two innovative products created: a standards guide, and educational software, which together provide a synthesis of the 'grammar' and the principles underlying the graphical definition of objects.

To date, no other reference work has achieved such an exhaustive, clear, and rigorous synthesis of these principles as the Standards Guide. It is vital that this knowledge be preserved in a form that can serve as a reference work for engineering students, whatever the future of graphical product definition may be, given that these are fundamental principles and independent of the distribution channel chosen.

Because of the nature of educational software and the way in which it is designed, this is a stand-alone product that can be used in all kinds of teaching and training situations: to complement a traditional course, as supplementary course material, and as a self-teaching tool that is accessible at all times. Its flexibility and longevity were, in fact, determining factors in the plan to include this software in the redesigned Bachelor of Engineering degree syllabus at Polytechnique Montréal.

We do not claim that these products are perfect. On the contrary, we regard them as being in a constant state of evolution. As such, comments and suggestions from students and teachers will continue to be considered, with the aim of improving their quality. Also, we wish to make it known that the products are available to other educational institutions, like universities and colleges.

With a view to improving the overall quality of teaching at Polytechnique Montréal, the methodology used in this research project (data synthesis and multimedia support with animations) could easily be applied to other courses in engineering curricula, to meet similar needs, notably those in which visual communication plays an important role.

\section{References}

[1] Canadian Standards Association. Dessins techniques principes généraux. Canadian Standards Association, 1984, 54 pp. CAN3-B78.1-M83.

[2] Canadian Standards Association. (1992). Cotation et tolérancement en dessin technique. Canadian Standards Association, 1992, 155 pp. CAN/CSA-B78.2-M91.

[3] Cincou, A. \& Riendeau, S.. Projection orthographique et visualisation spatiale. [Educational software]. Montréal, QC: École Polytechnique de Montréal, 2004. Available as of April 20, 2013, from http://www.moodle.polymtl.ca/

[4] Cincou, A. Guide des normes et conventions de représentation graphique. Montréal, QC: Presses internationales Polytechnique, 2011, 50 pp. \{ISBN: 978-2553-01598-4\}

[5] Giesecke, F.E., Mitchell, A., Spencer, H.C., Hill, I.L., \& Dygdon, J.T. Dessin technique. Saint-Laurent, QC: Éditions du renouveau pédagogique, 1982, 774 pp. \{ISBN: 2-76130041-6\}

[6] Giesecke, F.E., Mitchell, A., Spencer, H.C., Hill, I.L., \& Dygdon, J.T.. Dessin technique. Saint-Laurent, QC: Éditions du renouveau pédagogique, 1982 (special edition), 202 pp. \{ISBN: 2-3456-2234-2\}

[7] Giesecke, F.E., Mitchell, A., Spencer, H.C., Hill, I.L., \& Dygdon, J.T. Dessin technique. Saint-Laurent, QC: Éditions du renouveau pédagogique, 1982 (special edition, version 2), 238 pp. \{ISBN: 2-7613-1921-4\}

[8] International Organisation for Standardization. Dessins techniques : principes généraux de représentation. Partie 20. International Organization for Standardization, 1996 (1st ed.), 15 pp. ISO 128-20:1996 (F) 